\title{
Seven-Pass Transmembrane Cadherins: Roles and Emerging Mechanisms in Axonal and Dendritic Patterning
}

\author{
Sandra Berger-Müller • Takashi Suzuki
}

Received: 4 July 2011 / Accepted: 29 August 2011 / Published online: 10 September 2011

(C) The Author(s) 2011. This article is published with open access at Springerlink.com

\begin{abstract}
The Flamingo/Celsr seven-transmembrane cadherins represent a conserved subgroup of the cadherin superfamily involved in multiple aspects of development. In the developing nervous system, Fmi/Celsr control axonal blueprint and dendritic morphogenesis from invertebrates to mammals. As expected from their molecular structure, seven-transmembrane cadherins can induce cell-cell homophilic interactions but also intracellular signaling. Fmi/Celsr is known to regulate planar cell polarity (PCP) through interactions with PCP proteins. In the nervous system, Fmi/ Celsr can function in collaboration with or independently of other PCP genes. Here, we focus on recent studies which show that seven-transmembrane cadherins use distinct molecular mechanisms to achieve diverse functions in the development of the nervous system.
\end{abstract}

Keywords Flamingo $\cdot$ Celsr · Cadherins · GPCR-adhesion molecules · Dendrite formation · Axon guidance - Planar cell polarity · Golden goal

\section{Introduction}

Cadherins constitute a large family of more than 100 members that control diverse processes in development and act mainly through homophilic interactions [1, 2]. Cadherins are not just glue that maintains tight contacts between cells: in addition to mechanical adhesion, they mediate cell-cell communication and modulate cellular response

\footnotetext{
S. Berger-Müller · T. Suzuki $(\bowtie)$

Research Group Axon Guidance and Neuronal Connectivity,

Max Planck Institute of Neurobiology,

Am Kolpferspitz 18,

82152 Martinsried, Germany

e-mail: suzukit@neuro.mpg.de
}

through interactions with downstream intracellular components. Interestingly, in some cases, cadherins are able to activate signal transduction pathways even independently of homotypic adhesion [3].

$\mathrm{Fmi} / \mathrm{Celsr}$ is an evolutionary conserved atypical cadherin that contain a seven-pass transmembrane domain, a unique characteristic of this subfamily. The invertebrate Flamingo protein has three orthologs in mammals, named Celsr1-3 according to their structure (Cadherin EGF LAG Sevenpass G-type Receptor 1-3) [4-7].

$\mathrm{Fmi} / \mathrm{Celsr}$ is well known for regulating the establishment of planar cell polarity (PCP), a process that consists in the polarization of cells within the plane of the epithelium [811]. Fmi function has been extensively studied in the Drosophila wing, where it mediates homotypic interaction between adjacent cells and transmits instructive PCP signals $[4,5,12-14]$. In vertebrates, PCP phenotypes of Celsr mutants include the misorientation of hair cells $[15$, 16], the impairment of convergent extension [17] and defects in neural tube closure [15]. The core PCP genes Frizzled (Fz/Fzd), Van Gogh (Vang/Vang12), Dishevelled (Dsh/Dvl), and Prickle (Pk/Prkl) share phenotypic similarities with $\mathrm{Fmi} / \mathrm{Celsr}$ in all these aspects of development [18-21].

In addition to tissue/planar cell polarity, seventransmembrane cadherins have functions outside of the plane of the epithelium in the developing nervous system. A role for Fmi in the regulation of neurite morphogenesis and axon guidance has been first discovered in flies [2226], and these functions have been shown to be conserved in mammals [27-31]. Additionally, recent studies have highlighted a new role for Celsr in neuronal migration [3234].

Cadherins of the Fmi/Celsr subfamily are ambivalent proteins in that they have structural features of both cell 
adhesion molecules and signaling receptors (Fig. 1). Their conserved extracellular domain contains nine cadherins repeats known to mediate homophilic interactions, as well as EGF-like, laminin-G-like, and hormone receptor domains. Their seven-pass transmembrane domain is similar to G-protein-coupled receptors (GPCRs) of the secretin receptor family [35]. Fmi/Celsr also contains a GPS cleavage site next to the transmembrane domain characteristic of GPCR-adhesion molecules [36]. This complex protein structure suggests sophisticated mechanisms of seven-transmembrane cadherins that pose challenges to scientists trying to dissect their molecular functions. Although some mechanisms of action are emerging, they seem to be divergent in different contexts and remain elusive in many cases.

In this article, we will give an overview of the studies performed on $\mathrm{Fmi} / \mathrm{Celsr}$ in axon guidance and dendrite morphogenesis in different model organisms. We will emphasize the diversity of molecular strategies used by seven-transmembrane cadherins to control neuronal development, and discuss the different pathways in which they are involved.

\section{Fmi/Celsr in Axon Guidance and Dendritic Morphogenesis}

The formation of precise dendritic fields and the establishment of specific synaptic connections are crucial for proper sensory perception, brain-processing, and behavioral response. The development of neuronal processes and the regulation of their connections require directed guidance and cell-cell communication. Fmi/Celsr is involved in both axon guidance and dendritic patterning.

\section{Roles in Axon Pathfinding and Synaptic Targeting}

In the Drosophila visual system, Fmi regulates axon guidance and synaptic partner selection via axon-axon and axon-target interactions. During larval development, Fmi mediates competitive interactions between pioneer photoreceptor axons to maintain the axonal shafts at a proper distance from each other, thus ensuring the formation of a continuous topographic map (Fig. 2a) [25, 26]. During pupal development, photoreceptor axons that innervate the lamina defasciculate from their ommatidial bundle and extend in opposite directions to reach their proper post-synaptic partners (Fig. 2b). In fmi mutants, these photoreceptor axons choose inappropriate targets in the lamina [26], and Fmi was shown to act non-cellautonomously in this context [37]. By modulation Fmi expression level, Chen and colleagues revealed that Fmi homophilic interactions between adjacent unbundling growth cones mediate balanced forces that allow them to extend in the proper direction. In photoreceptors innervating the medulla, fmi mutant axons stop prematurely at the surface of the medulla $[25,38]$. The requirement of Fmi in both photoreceptor axons and their medulla target layer indicates that Fmi controls synaptic targeting by homophilic axontarget interactions in the medulla [38]. In addition, fmi mutants show an axon stalling phenotype in abdominal sensory neurons and motor neurons in the embryo [39], suggesting that Fmi is widely implicated in axon pathfinding in Drosophila.

Recently, it was shown that fmi plays a role in axon guidance in the early development of the ventral nerve cord in Caenorhabditis elegans [40]. Fmi is required in both pioneer and follower axons, indicating distinct functions in axon pathfinding and axon fasciculation.
Fig. 1 Molecular structure of Fmi/Celsr family members. Flamingo and Celsr extracellular domains comprises nine cadherin repeats, a series of EGFlike and laminin globular-like domains, and a hormone receptor domain (HRM). The seventransmembrane domain, similar to those found in GPCRs, is characteristic of this atypical cadherin subfamily. The GPCR proteolytic site (GPS) is conserved in many adhesion GPCRs. The intracellular domain is generally not conserved and does not contain recognizable domains. Adapted from [2]

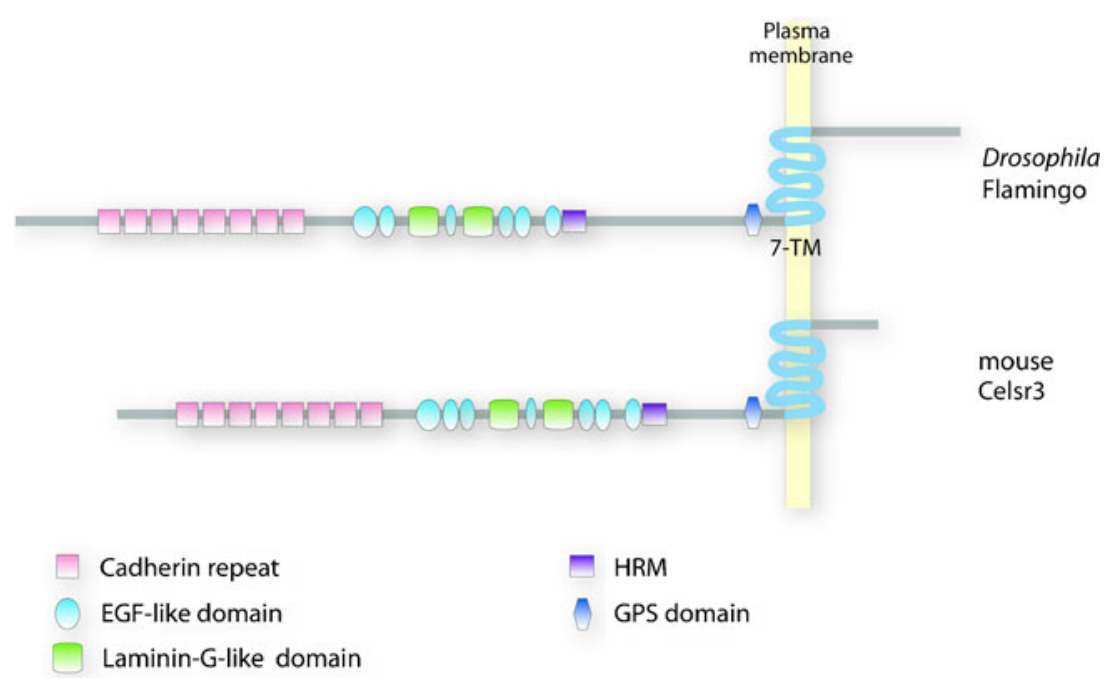


a

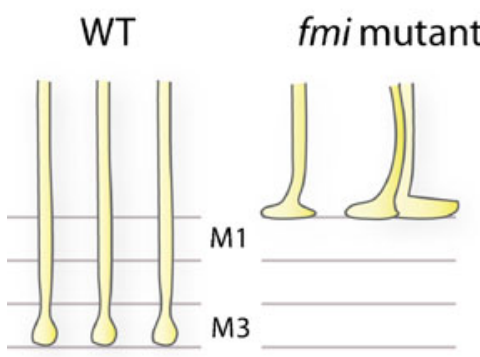

C

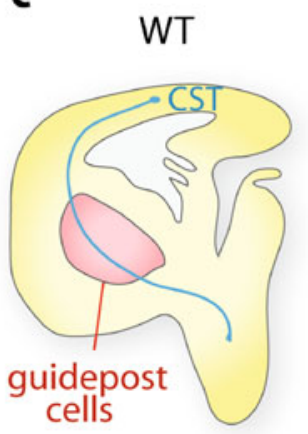

Fig. 2 Examples of Fmi/Celsr phenotypes in axon guidance and targeting. a In the Drosophila visual system, R8 photoreceptor axons are arranged in evenly spaced topographic arrays and target the M3 layer in the medulla. In fmi mutants, competitive axon-axon interactions are lost leading to an axon bundling phenotype. Moreover, R8 axons stop prematurely at the M1 layer due to impaired Fmi homophilic axon-target interactions. b Photoreceptor axons arrive in the fly lamina as an ommatidial bundle. Axons defasciculate and grow b

WT

fmi mutant
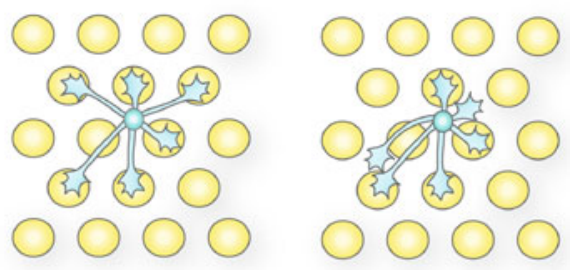

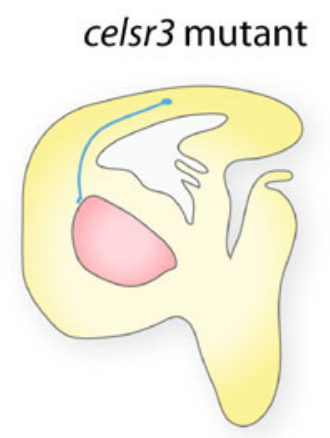

perpendicularly to the bundle in stereotyped directions to reach their correct target. In fmi mutants, axons make directional errors and innervate inappropriate targets. Adapted from [2]. c In mice, several axon tracts of the internal capsule are misguided in Celsr 3 mutants, including subcerebral projections (CST, blue). These tracts are defective when Celsr3 is absent in these axons or in guidepost cell (red area), suggesting that Celsr3 regulates axon guidance via homophilic interactions. Adapted from [31]

\section{Regulation of Dendritic Patterning}

in axonal blueprint in the mammalian central nervous system. Celsr 3 mutant mice have severe defects in several major tracts including the anterior commissure and the internal capsule [29]. Using several conditional knock-out mice, Zhou and colleagues demonstrated that Celsr 3 acts cell-autonomously in neurons forming these axonal tracts, but in the internal capsule, Celsr 3 is also required in cells located on their trajectory (Fig. 2c) [41]. This indicates that Celsr3 regulates axon pathfinding via homophilic interactions between axons and guidepost cells. Additionally, Celsr3 was shown to guide axons in the mice spinal cord: instead of turning anteriorly, mutant commissural axons extend randomly along the anterior-posterior axis [18, 30, 42]. Similarly, Celsr 3 mutant mice show anterior-posterior guidance defects of serotonergic and dopaminergic neurons in the brainstem [43].

Besides its role in axon guidance, Fmi have additional functions as a negative regulator of synaptogenesis and axonal degeneration in Drosophila motorneurons [44].

In the fly peripheral nervous system, Fmi is involved is two distinct developmental steps of dendritic field formation. During embryonic development, dendrites start to grow toward the midline, and pause before reaching it in wildtype animals. Dendritic growth restarts during larval stages: dendrites from two contralateral hemisegments meet at the midline and repel each other, leading to the formation of non-overlapping dendritic fields. In fmi mutants, however, dendrites grow precociously and cross the midline in the embryo [22]. In fmi mutant larvae, dendrites lose competition between homologous neurons and invade the contralateral segment (Fig. 3a) [45]. A role for Fmi in the repression of dendritic growth has been reported also in mushroom body neurons [24], indicating that Fmi is a general negative regulator of dendritic extension in the central and peripheral nervous system in the fly.

Interestingly, in the mammalian nervous system, Celsr 2 and Celsr 3 regulate neurite arborization in opposite ways. Using gene silencing in rat neuronal cultures, Shima et al. 
a

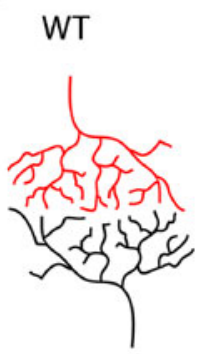

b

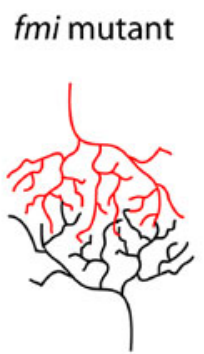

control

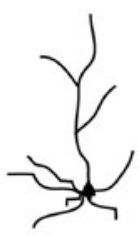

Celsr2 knockdown

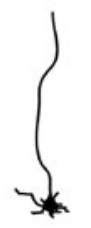

Celsr3

knockdown

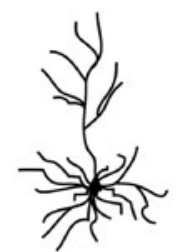

Fig. 3 Roles of Fmi/Celsr in dendritic morphogenesis. a In Drosophila melanogaster larvae, dendrites of peripheral neurons cover their entire sensory field but always avoid dendrites of homologous neurons in the contralateral side. In fmi mutants,

showed that Celsr 2 promotes dendritic extension, whereas Celsr3 represses it (Fig. 3b) [27, 28]. Co-culture experiments showed that Celsr2 and Celsr3 trigger neurite growth or retraction through homophilic interactions. This indicates that Celsr homotypic interactions at dendrodendritic contacts may be involved in dendritic patterning.

\section{Fmi/Celsr: Simple Adhesion Molecules or Signaling Receptors?}

The molecular structure of seven-transmembrane cadherins suggests that they can mediate cell-cell adhesion and signaling functions. Several experiments support the idea that Fmi/Celsr is involved in homophilic adhesion. First, in vitro, the expression of Fmi or Celsr2 in Drosophila S2 cells lead to the formation of cell aggregates, an effect which is dependent on the cadherin repeats [5, 27, 45]. Second, in the Drosophila visual system, Fmi mediates homophilic interactions between photoreceptor axons and their target in the medulla, thus allowing the recognition and adhesion of pre- and postsynaptic partners [38]. In this process, Fmi intracellular domain is not required in photoreceptor axons, but we cannot completely exclude that Fmi signals via its sevenpass transmembrane domain. Additionally, in the ventral nerve cord of $C$. elegans, fmi mutant follower axons show a defasciculation phenotype, indicating that Fmi mediates adhesion between pioneer and follower axons [40]. Notably, neither the intracellular nor the seventransmembrane domain are required for the follower axons to fasciculate with the pioneer axons, indicating that Fmi could act as a pure adhesive factor in this case. Another example that argues for homophilic adhesion is that Celsr3 is required in both navigating axons and guidepost cells in the internal capsule in mammals [41].

Even though Fmi/Celsr seems to act homophilically and adhesively in several cases, some evidence indicates however, dendrodendritic competitive interactions are lost, and dendrites invade the contralateral hemisegment. b In rat neuronal cultures, knocking down Celsr 2 or Celsr 3 suppresses or enhances neurite growth, respectively

that they can also elicit downstream signaling. In the ventral nerve cord of $C$. elegans for instance, the intracellular domain of Fmi is crucial for guiding pioneer axons (as opposed to follower axons), suggesting that interactions with intracellular components is important for axon pathfinding [40]. Intriguingly, the dendritic overgrowth phenotype in Drosophila fmi mutant embryo can be partially rescued by a Fmi construct lacking the cadherin repeats and the EGF/laminin domains (but retains the HRM domain) [45]. The same construct is also able to partially rescue the axon stalling phenotype in Drosophila embryonic sensory neurons [39]. These results suggest that Fmi can transmit a signal independently of homophilic cell adhesion, and indicates that either Fmi binds to an unknown ligand or is part of a signaling protein complex. Finally, the most compelling evidence that seven-transmembrane cadherins can induce intracellular signaling comes from the study by Shima et al. [28], in which they demonstrate that Celsr2 and Celsr3 trigger intracellular $\mathrm{Ca}^{2+}$ increase upon binding to their respective cadherin repeats. It remains to be confirmed that Celsr2,3 are real GPCRs by identifying the G-protein they activate.

Notably, even when Fmi does not elicit downstream signaling by itself, it can do so via a cis complex: in Drosophila, the axon guidance response is mainly mediated by the intracellular domain of the cell-surface molecule Golden Goal (Gogo) [38] (see below).

In summary, Fmi/Celsr seems to act homophilically in many cases, but also often transmit an intracellular signal in axons and dendrites.

\section{Attraction vs. Repulsion}

Homophilic binding can mediate cell adhesion as described above. However, cell-cell interactions can also trigger signaling cascades that lead to neurite advance or retraction. The outcome of Fmi/Celsr homophilic interactions is not 
always clear and seems to be context dependent. In the Drosophila visual system, Fmi interactions induce balanced forces between defasciculating axons in the lamina to control their extension towards their proper targets [37]. However, it is not known whether Fmi mediates repulsive or attractive interactions between unbundling growth cones. In axon targeting in the fly medulla, fmi mutants photoreceptor axons fail to extend from their temporary target to their final synaptic-layer. This indicates that Fmi serves as a pavement on the path to pull axons toward their final destination. In the mammalian nervous system, Celsr3 could act in a similar way since the removal of Celsr3 in guidepost cells induces the stopping of subcerebral tracts [41].

On the contrary, the fmi mutant dendritic phenotype in Drosophila suggests that Fmi is involved in neurite retraction or repression of growth [22, 24, 45]. Repulsion seems to be mediated by different molecular mechanisms at different stages of development. In the embryo, dendrites migrate towards the contralateral segment but never touch the dendrites of homologous neurons, and Fmi lacking the cadherin repeats can partially rescue the fmi mutant phenotype, suggesting that dendritic repulsion is not the result of homophilic binding. On the contrary, in larvae, Fmi mediates competitive interactions between homologous neurons to shape their dendritic fields [24], and its cadherin repeats are required for dendritic tiling [45], indicating that Fmi homophilic interactions at dendrodendritic contacts induces repulsion.

In mammals, the functions of Celsr2 and Celsr3 in dendrite morphogenesis have diverged. Celsr2 induces the formation of longer dendrites with complex arborization, whereas Celsr3 suppresses neurite growth [28]. A single amino acid in the first loop of the intracellular domain is responsible for these opposite roles: exchanging the histidine residue of Celsr3 with arginin (present in Celsr2) generates Celsr2-like effects on neurites and vice-versa. Like Celsr3, Fmi possess a histidine at this position, consistent with its repulsive role in dendritic field formation.

\section{Fmi/Celsr Uses Distinct Signaling Pathways}

Fmi/Celsr acts in the PCP pathway in different aspects of development, including hair cell polarization, convergent extension, neural tube closure, and neuronal migration. Several PCP genes are involved in axonal blueprint in mammals and flies, even if the pathways involved may not be strictly identical as the one regulating PCP events. Fmi/Celsr also functions in PCP-independent pathways in axon guidance and dendritic morphogenesis.
PCP Genes in Neuronal Connections and Morphogenesis

Fzd3 mutant mice have similar phenotypes to Celsr3 mutants in brain wiring, including defects in the anterior commissure and the internal capsule [46]. Together with the fact that $\mathrm{Fzd} 3$ and $\mathrm{Celsr} 3$ have an overlapping expression pattern in developing neurons [29], it provides strong evidence that these two genes interact during the establishment of axonal blueprint, maybe in collaboration with other PCP genes.

In the mice spinal cord, Celsr3, Fzd3, and Vangl2 mutants all show similar guidance defects along the anterior-posterior axis in post-crossing commissural axons $[30,47]$ and Wnts gradients attract these axons anteriorly $[47,48]$. Shafer et al. unveiled an antagonistic interaction between Dvl1 and Vangl2 to regulate Fzd3 signaling. Similarly, Celsr3, Fzd3, and Vangl2 mutants have guidance defects in the anterior-posterior axis in dopaminergic neurons in the midbrain and serotonergic neurons in the hindbrain. Wnt $5 \mathrm{~A}$ mutants also show anterior-posterior guidance errors in dopaminergic projections [43]. It would be interesting to know if Celsr3 functions in axons by regulating the growth cone localization or signaling of $\mathrm{PCP}$ components, or by homophilic interactions with neighboring cells.

In Drosophila, it was recently shown that the PCP genes $f z, d s h$, vang, and wnt5 are involved and cooperate in the targeting and branching of mushroom body neurons ([49]). Knocking down fmi in these neurons generates a similar phenotype. It would be interesting to further investigate the role of fmi and its interaction with other PCP molecules in this process.

Overall, PCP genes are acting in various aspects of development and can mediate directed movement of cells (convergent extension and neuronal migration) and of growth cones. The next challenge is to explore the similarities and/or differences in the pathways involved in pure PCP events and in neurite development.

\section{PCP-Independent Pathways}

Unlike in mice, Fmi can act in a PCP-independent pathway in axon guidance in Drosophila. The PCP mutants for $f z$, vang, $d s h$, and $p k$ show completely normal axon targeting of photoreceptors in the medulla [38]. Similarly, fz, vang, and $d s h$ mutants do not display the axon stalling phenotype seen in fmi mutant sensory neurons [39].

In photoreceptor axon targeting, the transmembrane receptor Golden Goal (Gogo) has been recently identified as a molecular partner of Fmi [38, 50, 51]. gogo shares striking phenotypic similarities and genetically interacts with fmi in target selection of photoreceptor cells and in dendritic growth. In addition, Gogo and Fmi can mutually 
influence their localization. Fmi seems to trigger intracellular signaling via Gogo, since the synergistic effect of Gogo and Fmi overexpressions on photoreceptor targeting depends on Gogo cytoplasmic domain. Interestingly, Fmi is crucial in the target area for photoreceptor targeting, whereas Gogo is not required in target cells, suggesting that like in PCP, homophilic asymmetric interactions governs axon-target recognition, which may be a general mechanism of action for Fmi. Gogo is a structurally conserved protein, yet a neuronal function for the mouse ortholog Tmtsp has not been described so far [52].

In dendrite morphogenesis, Fmi/Celsr seems to be involved in other signaling pathway than the PCP pathway. Unlike fmi mutants, $f z$ mutant embryos have a wild-type phenotype in dendritic development of peripheral neurons in Drosophila [22]. In mammals, Celsr2,3 seem to work in a PCP-independent pathway in dendritic growth and arborization, since they act via a second messenger, maybe as a GPCR [28].

The fact that Fmi is involved in different pathways raises the question of how interactions between different partners at distinct subcellular locations are coordinated in single cells. For example, Drosophila photoreceptor neurons undergo both cell body polarization via the PCP pathway and axon guidance through Gogo. In this case, it seems that a tight regulation of gene expression allows the separation of Fmi functions, since precocious Gogo expression during the establishment of PCP induces ommatidial orientation defects (our unpublished data).

\section{Concluding Remarks}

To achieve their multiple functions in development, sevenpass transmembrane cadherins use diverse molecular mechanisms: depending on the context, they act in a cell autonomous or non-cell autonomous fashion, have different domain requirements, mediate adhesive or repulsive interactions, and function through distinct molecular pathways (Fig. 4).

It seems that Fmi/Celsr works together with PCP genes in development processes that require cell polarity, including hair cell orientation, convergence and extension, neuronal migration, and growth cone turning. In contrast, Fmi/Celsr may act with other partners in dendritogenesis, axon advance, and axon fasciculation/defasciculation.

Mutant analyses suggest that there is a functional separation between mice Celsr1 and Celsr2,3. Celsr1 is
Fig. 4 Models of the diverse molecular mechanisms of seventransmembrane cadherins in neuronal interactions and connections. a Fmi/Celsr could act as pure adhesive molecules, without transmitting an intracellular signal. b Fmi/Celsr may be a receptor for an unknown ligand. c In mammals, Celsr2 and Celsr3 homotypic interactions trigger intracellular calcium increase, leading to opposite responses in dendritogenesis. d Fzd3 collaborates with Celsr3 in the formation of axonal tracts in mammals. The other PCP proteins Vang12, Dvl, and Wnts are also involved in the guidance of commissural axons in the mice spinal cord. e In the fly visual system, synaptic-layer targeting in the medulla is mediated by asymmetric homophilic interaction, involving Fmi and Gogo in photoreceptors and Fmi in their target. Gogo interacts with the cytoskeleton via Adducin
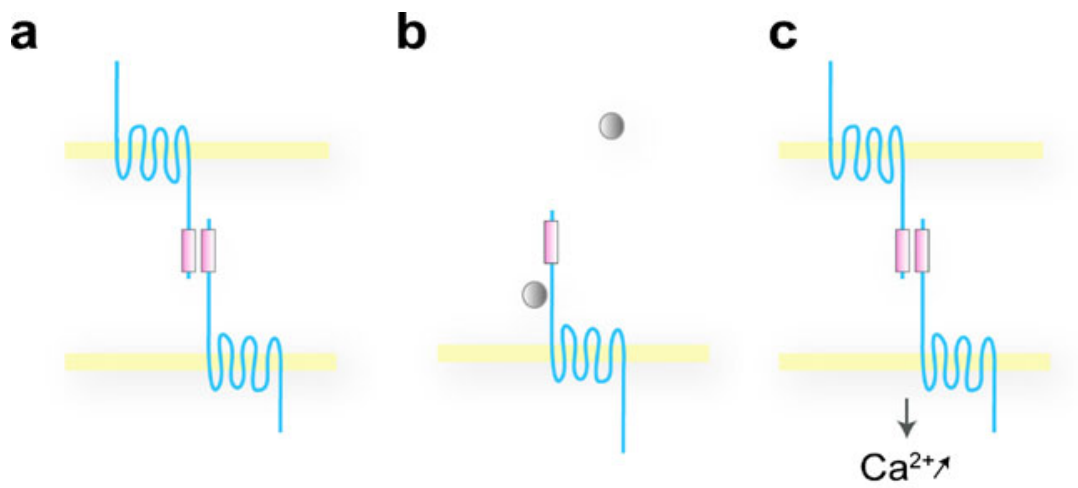

simple adhesion unknown ligand second messenger
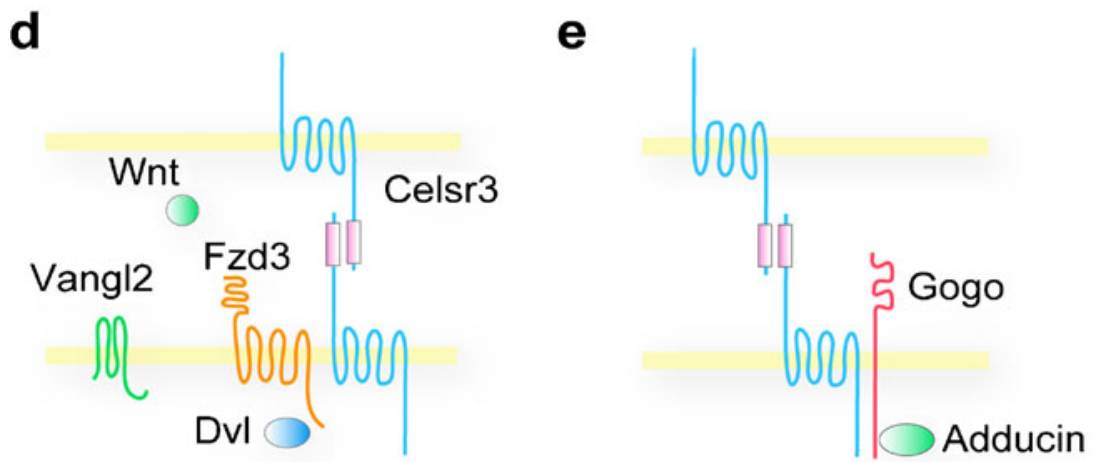

PCP pathway 
involved in pure PCP events, like hair cell orientation in inner ear cells and neural tube closure [15]. In contrast, Celsr 2,3 function in non-planar and non-epithelial processes like axonal tract development and dendritic patterning. Additionally, even if all Celsr 1-3 regulate facial branchiomotor (FBM) neurons migration, Celsr 1 and Celsr 3 have different phenotypes and use different mechanisms, whereas Celsr2 and Celsr3 seem to be redundant [34]. The complementary expression pattern of Celsr genes, mostly in precursor neurons for Celsr1, and mainly in post-mitotic neurons for Celsr2,3 further supports the idea that Celsr 1 and Celsr 2,3 have divergent functions in mammals [20, 5356]. Therefore, Celsr appear to be an example of gene duplication-degeneration-complementation, a process by which duplicated genes fulfill the roles of the initial ancestor gene in a complementary manner [57]. This picture may be different in Zebrafish, where Celsrl and Celsr2 possibly have redundant functions in convergent extension [17] and migration of FBM neurons [32].

To conclude, the intense investigation of Fmi/Celsr function in diverse developmental aspects demonstrated that multiple molecular mechanisms and pathways are at play. Although we are far from completely understanding how seven-transmembrane cadherins regulate neuronal development, what has emerged so far from the different studies is that Fmi/Celsr mainly acts homophilically, mediates both adhesion/attraction and repulsion, and acts in different pathways depending on the context. Further structure-function studies and the identification of interacting partners in each particular context will help to unveil the mechanisms of action of these atypical cadherins.

Open Access This article is distributed under the terms of the Creative Commons Attribution Noncommercial License which permits any noncommercial use, distribution, and reproduction in any medium, provided the original author(s) and source are credited.

\section{References}

1. Halbleib JM, Nelson WJ (2006) Cadherins in development: cell adhesion, sorting, and tissue morphogenesis. Genes Dev 20:31993214

2. Takeichi M (2007) The cadherin superfamily in neuronal connections and interactions. Nat Rev Neurosci 8:11-20

3. Cavallaro U, Dejana E (2011) Adhesion molecule signalling: not always a sticky business. Nat Rev Mol Cell Biol 12:189-197

4. Chae J, Kim MJ, Goo JH, Collier S, Gubb D, Charlton J, Adler PN, Park WJ (1999) The Drosophila tissue polarity gene starry night encodes a member of the protocadherin family. Development 126:5421-5429

5. Usui T, Shima Y, Shimada Y, Hirano S, Burgess RW, Schwarz TL, Takeichi M, Uemura T (1999) Flamingo, a seven-pass transmembrane cadherin, regulates planar cell polarity under the control of Frizzled. Cell 98:585-595

6. Hadjantonakis AK, Sheward WJ, Harmar AJ, de Galan L, Hoovers JM, Little PF (1997) Celsr1, a neural-specific gene encoding an unusual seven-pass transmembrane receptor, maps to mouse chromosome 15 and human chromosome 22qter. Genomics 45:97-104

7. Formstone CJ, Barclay J, Rees M, Little PF (2000) Chromosomal localization of Celsr2 and Celsr3 in the mouse; Celsr3 is a candidate for the tippy (tip) lethal mutant on chromosome 9. Mamm Genome 11:392-394

8. Goodrich LV (2008) The plane facts of PCP in the CNS. Neuron 60:9-16

9. Simons M, Mlodzik M (2008) Planar cell polarity signaling: from fly development to human disease. Annu Rev Genet 42:517-540

10. Klein TJ, Mlodzik M (2005) Planar cell polarization: an emerging model points in the right direction. Annu Rev Cell Dev Biol 21:155-176

11. Zallen JA (2007) Planar polarity and tissue morphogenesis. Cell 129:1051-1063

12. Strutt H, Strutt D (2008) Differential stability of flamingo protein complexes underlies the establishment of planar polarity. Curr Biol 18:1555-1564

13. Chen WS, Antic D, Matis M, Logan CY, Povelones M, Anderson GA, Nusse R, Axelrod JD (2008) Asymmetric homotypic interactions of the atypical cadherin flamingo mediate intercellular polarity signaling. Cell 133:1093-1105

14. Shimada Y, Usui T, Yanagawa S, Takeichi M, Uemura T (2001) Asymmetric colocalization of Flamingo, a seven-pass transmembrane cadherin, and Dishevelled in planar cell polarization. Curr Biol 11:859-863

15. Curtin JA, Quint E, Tsipouri V, Arkell RM, Cattanach B, Copp AJ, Henderson DJ, Spurr N, Stanier P, Fisher EM, Nolan PM, Steel KP, Brown SD, Gray IC, Murdoch JN (2003) Mutation of Celsr1 disrupts planar polarity of inner ear hair cells and causes severe neural tube defects in the mouse. Curr Biol 13:1129-1133

16. Devenport D, Fuchs E (2008) Planar polarization in embryonic epidermis orchestrates global asymmetric morphogenesis of hair follicles. Nat Cell Biol 10:1257-1268

17. Formstone CJ, Mason I (2005) Combinatorial activity of Flamingo proteins directs convergence and extension within the early zebrafish embryo via the planar cell polarity pathway. Dev Biol 282:320-335

18. Wang Y, Nathans J (2007) Tissue/planar cell polarity in vertebrates: new insights and new questions. Development 134:647-658

19. Wada H, Okamoto H (2009) Roles of planar cell polarity pathway genes for neural migration and differentiation. Dev Growth Differ 51:233-240

20. Tissir F, Goffinet AM (2010) Planar cell polarity signaling in neural development. Curr Opin Neurobiol 20:572-577

21. Wada H, Okamoto H (2009) Roles of noncanonical Wnt/PCP pathway genes in neuronal migration and neurulation in zebrafish. Zebrafish 6:3-8

22. Gao FB, Kohwi M, Brenman JE, Jan LY, Jan YN (2000) Control of dendritic field formation in Drosophila: the roles of flamingo and competition between homologous neurons. Neuron 28:91-101

23. Sweeney NT, Li W, Gao FB (2002) Genetic manipulation of single neurons in vivo reveals specific roles of flamingo in neuronal morphogenesis. Dev Biol 247:76-88

24. Reuter JE, Nardine TM, Penton A, Billuart P, Scott EK, Usui T, Uemura T, Luo L (2003) A mosaic genetic screen for genes necessary for Drosophila mushroom body neuronal morphogenesis. Development 130:1203-1213

25. Senti KA, Usui T, Boucke K, Greber U, Uemura T, Dickson BJ (2003) Flamingo regulates R8 axon-axon and axon-target interactions in the Drosophila visual system. Curr Biol 13:828832

26. Lee RC, Clandinin TR, Lee CH, Chen PL, Meinertzhagen IA, Zipursky SL (2003) The protocadherin Flamingo is required for 
axon target selection in the Drosophila visual system. Nat Neurosci 6:557-563

27. Shima Y, Kengaku M, Hirano T, Takeichi M, Uemura T (2004) Regulation of dendritic maintenance and growth by a mammalian 7-pass transmembrane cadherin. Dev Cell 7:205-216

28. Shima Y, Kawaguchi SY, Kosaka K, Nakayama M, Hoshino M, Nabeshima Y, Hirano T, Uemura T (2007) Opposing roles in neurite growth control by two seven-pass transmembrane cadherins. Nat Neurosci 10:963-969

29. Tissir F, Bar I, Jossin Y, De Backer O, Goffinet AM (2005) Protocadherin Celsr3 is crucial in axonal tract development. Nat Neurosci 8:451-457

30. Shafer B, Onishi K, Lo C, Colakoglu G, Zou Y (2011) Vang12 promotes Wnt/planar cell polarity-like signaling by antagonizing Dvl1-mediated feedback inhibition in growth cone guidance. Dev Cell 20:177-191

31. Zhou L, Qu Y, Tissir F, Goffinet AM (2009) Role of the atypical cadherin Celsr3 during development of the internal capsule. Cereb Cortex 19(Suppl 1):i114-i119

32. Wada H, Tanaka H, Nakayama S, Iwasaki M, Okamoto H (2006) Frizzled3a and Celsr2 function in the neuroepithelium to regulate migration of facial motor neurons in the developing zebrafish hindbrain. Development 133:4749-4759

33. Ying G, Wu S, Hou R, Huang W, Capecchi MR, Wu Q (2009) The protocadherin gene Celsr3 is required for interneuron migration in the mouse forebrain. Mol Cell Biol 29:3045-3061

34. Qu Y, Glasco DM, Zhou L, Sawant A, Ravni A, Fritzsch B, Damrau C, Murdoch JN, Evans S, Pfaff SL, Formstone C, Goffinet AM, Chandrasekhar A, Tissir F (2010) Atypical cadherins Celsr1-3 differentially regulate migration of facial branchiomotor neurons in mice. J Neurosci 30:9392-9401

35. Harmar AJ (2001) Family-B G-protein-coupled receptors. Genome Biol 2:REVIEWS3013.

36. Lin HH, Stacey M, Yona S, Chang GW (2011) GPS proteolytic cleavage of adhesion-GPCRs. Adv Exp Med Biol 706:49-58

37. Chen PL, Clandinin TR (2008) The cadherin Flamingo mediates level-dependent interactions that guide photoreceptor target choice in Drosophila. Neuron 58:26-33

38. Hakeda-Suzuki S, Berger-Muller S, Tomasi T, Usui T, Horiuchi SY, Uemura T, Suzuki T (2011) Golden Goal collaborates with Flamingo in conferring synaptic-layer specificity in the visual system. Nat Neurosci 14:314-323

39. Steinel MC, Whitington PM (2009) The atypical cadherin Flamingo is required for sensory axon advance beyond intermediate target cells. Dev Biol 327:447-457

40. Steimel A, Wong L, Najarro EH, Ackley BD, Garriga G, Hutter H (2010) The Flamingo ortholog FMI-1 controls pioneer-dependent navigation of follower axons in $C$. elegans. Development 137:3663-3673

41. Zhou L, Bar I, Achouri Y, Campbell K, De Backer O, Hebert JM, Jones K, Kessaris N, de Rouvroit CL, O'Leary D, Richardson WD, Goffinet AM, Tissir F (2008) Early forebrain wiring: genetic dissection using conditional Celsr3 mutant mice. Science 320:946-949

42. Price DJ, Kennedy H, Dehay C, Zhou L, Mercier M, Jossin Y, Goffinet AM, Tissir F, Blakey D, Molnar Z (2006) The development of cortical connections. Eur J Neurosci 23:910-920
43. Fenstermaker AG, Prasad AA, Bechara A, Adolfs Y, Tissir F, Goffinet A, Zou Y, Pasterkamp RJ (2010) Wnt/planar cell polarity signaling controls the anterior-posterior organization of monoaminergic axons in the brainstem. J Neurosci 30:16053-16064

44. Bao H, Berlanga ML, Xue M, Hapip SM, Daniels RW, Mendenhall JM, Alcantara AA, Zhang B (2007) The atypical cadherin flamingo regulates synaptogenesis and helps prevent axonal and synaptic degeneration in Drosophila. Mol Cell Neurosci 34:662-678

45. Kimura H, Usui T, Tsubouchi A, Uemura T (2006) Potential dual molecular interaction of the Drosophila 7-pass transmembrane cadherin Flamingo in dendritic morphogenesis. J Cell Sci 119:1118-1129

46. Wang Y, Thekdi N, Smallwood PM, Macke JP, Nathans J (2002) Frizzled-3 is required for the development of major fiber tracts in the rostral CNS. J Neurosci 22:8563-8573

47. Lyuksyutova AI, Lu CC, Milanesio N, King LA, Guo N, Wang Y, Nathans J, Tessier-Lavigne M, Zou Y (2003) Anterior-posterior guidance of commissural axons by Wnt-frizzled signaling. Science 302:1984-1988

48. Wolf AM, Lyuksyutova AI, Fenstermaker AG, Shafer B, Lo CG, Zou Y (2008) Phosphatidylinositol-3-kinase-atypical protein kinase $\mathrm{C}$ signaling is required for Wnt attraction and anteriorposterior axon guidance. J Neurosci 28:3456-3467

49. Shimizu K, Sato M, Tabata T (2011) The Wnt5/planar cell polarity pathway regulates axonal development of the Drosophila mushroom body neuron. J Neurosci 31:4944-4954

50. Tomasi T, Hakeda-Suzuki S, Ohler S, Schleiffer A, Suzuki T (2008) The transmembrane protein Golden goal regulates R8 photoreceptor axon-axon and axon-target interactions. Neuron 57:691-704

51. Ohler S, Hakeda-Suzuki S, Suzuki T (2011) Hts, the Drosophila homologue of Adducin, physically interacts with the transmembrane receptor Golden goal to guide photoreceptor axons. Dev Dyn 240:135-148

52. Takayanagi S, Hiroyama T, Yamazaki S, Nakajima T, Morita Y, Usui J, Eto K, Motohashi T, Shiomi K, Keino-Masu K, Masu M, Oike Y, Mori S, Yoshida N, Iwama A, Nakauchi H (2006) Genetic marking of hematopoietic stem and endothelial cells: identification of the Tmtsp gene encoding a novel cell surface protein with the thrombospondin-1 domain. Blood 107:4317-4325

53. Tissir F, Goffinet AM (2006) Expression of planar cell polarity genes during development of the mouse CNS. Eur J Neurosci 23:597-607

54. Tissir F, De-Backer O, Goffinet AM, Lambert de Rouvroit C (2002) Developmental expression profiles of Celsr (Flamingo) genes in the mouse. Mech Dev 112:157-160

55. Shima Y, Copeland NG, Gilbert DJ, Jenkins NA, Chisaka O, Takeichi M, Uemura T (2002) Differential expression of the sevenpass transmembrane cadherin genes Celsr1-3 and distribution of the Celsr2 protein during mouse development. Dev Dyn 223:321-332

56. Formstone CJ, Little PF (2001) The flamingo-related mouse Celsr family (Celsr1-3) genes exhibit distinct patterns of expression during embryonic development. Mech Dev 109:91-94

57. Force A, Lynch M, Pickett FB, Amores A, Yan YL, Postlethwait J (1999) Preservation of duplicate genes by complementary, degenerative mutations. Genetics 151:1531-1545 